\title{
Health inequalities
}

G. McCartney, F. Popham, R. McMaster, A. Cumbers

\section{Source}

G. McCartney, F. Popham, R. McMaster, A. Cumbers. (2019). Defining health and health inequalities. Public Health, vol. 172, 22-30. doi:10.1016/j.puhe.2019.03.023.

Health inequalities are the systematic, avoidable and unfair differences in health outcomes that can be observed between populations, between social groups within the same population or as a gradient across a population ranked by social position. 